\title{
Investigação dos teores de metais pesados em espécies de siris azuis do gênero Callinectes sp.
}

\author{
Heavy metals content investigation in blue crab species of the genus Callinectes sp.
}

\author{
Rossana Helena Pitta VIRGA ${ }^{1}$, Luiz Paulo GERALDO ${ }^{1 \star}$
}

\begin{abstract}
Resumo
A ocorrência de metais pesados em sistemas aquáticos, usualmente, é resultante de processos naturais geoquímicos. Entretanto, a presença destes elementos químicos tem aumentado consideravelmente nas últimas décadas, como no caso da bacia do rio Cubatão, em conseqüência das atividades humanas na região. Neste trabalho, foi realizada uma análise quantitativa do conteúdo de $\mathrm{Pb}, \mathrm{Cd}, \mathrm{Cr}, \mathrm{Zn}$ e Cu, em quatro espécies de siris azuis do gênero Callinectes sp., coletadas ao longo do rio Cubatão, utilizando a técnica da espectrometria de absorção atômica por chama (FAAS). Foram analisadas 144 amostras de siris azuis das seguintes espécies: C. danae (63\%); C. sapidus (23\%); C. bocourti (10.5\%); e C. ornatus (3.5\%). A espécie C. Sapidus foi a que apresentou os maiores níveis de concentração para os metais estudados. Com exceção do $\mathrm{Cu}$, cuja concentração média obtida foi $40 \%$ maior para as fêmeas ( $\mathrm{p}<0,05)$, não houve qualquer variação significativa para os teores de metais pesados em relação tanto ao sexo quanto ao tamanho dos animais. Para o Cr, o intervalo de valores médios obtidos para as 4 espécies ficou acima do limite permitido pela legislação brasileira para qualquer tipo de alimento. Todos os outros resultados estiveram abaixo dos valores máximos recomendados pelas agências de saúde.
\end{abstract}

Palavras-chave: metal pesado; contaminação; siri azul.

\begin{abstract}
Usually, heavy metals occurrence in aquatic systems is resulting from natural geochemical processes. However, the presence of these chemical elements has considerably increased in the last decades. An example is the case of the Cubatão river basin caused by human activities in that region. In this work, a quantitative analysis of $\mathrm{Pb}, \mathrm{Cd}, \mathrm{Cr}, \mathrm{Zn}$, and $\mathrm{Cu}$ content of four blue crab species of the genus Callinectes sp., sampled along the Cubatão river, was carried out using a flame atomic absorption spectrometer (FAAS). 144 blue crab samples from the following species were analyzed: C. danae (63\%), C. sapidus (23\%), C. bocourti (10.5\%), and C. ornatus (3.5\%). The C. Sapidus species presented the highest concentration levels for the studied metals. With the exception of $\mathrm{Cu}$, for which the average concentration was $40 \%$ higher for the females $(\mathrm{p}<0,05)$, there was no significant variation in the heavy metal content regarding sex and the size of the animals. Except for Cr, for which the average values interval obtained for the 4 species was above the limit allowed by the Brazilian legislation for any type of food, all the other results were below the maximum values recommended by health agencies.

Keywords: heavy metal; contamination; blue crab.
\end{abstract}

\section{Introdução}

Um dos aspectos críticos da introdução de substâncias químicas em sistemas aquáticos é a sua bioacumulação na cadeia alimentar existente nestes compartimentos ambientais, principalmente, no que se refere à contaminação por metais pesados (MARKERT, 1998).

No município de Cubatão foram instaladas, a partir da década de 1950, importantes indústrias petroquímicas, metalúrgicas, fertilizantes, entre outras, aproveitando-se das facilidades do maior porto marítimo do País, o Porto de Santos. O material poluente lançado na região por essas indústrias levou a um processo de degradação ambiental intenso na região.

Na década de 1980, a CETESB-Companhia de Tecnologia de Saneamento Ambiental (SP) implantou um programa rígido de controle de poluição ambiental no município de Cubatão, o que reduziu drasticamente a ocorrência de episódios críticos de poluição do ar e tornou possível a recuperação da qua- lidade das águas. Contudo, estudos realizados pela própria CETESB (1990) utilizando amostras de águas, sedimentos e organismos aquáticos provenientes da bacia do rio Cubatão, constataram ainda a presença de alguns metais pesados e compostos orgânicos em concentrações muitas vezes superiores aos limites recomendados pela WHO (1972). No último relatório publicado pela CETESB (2001), os resultados mostraram uma redução (maior que 50\%) da contaminação nos organismos, em relação aos estudos anteriores, para alguns metais (cádmio, chumbo, mercúrio) e alguns compostos orgânicos (hexaclorobenzeno). Como conseqüência, observou-se o retorno de peixes e outros organismos aquáticos, que haviam emigrados da bacia do rio Cubatão. Entretanto, esta transformação ambiental na região não garante a ocorrência de uma recuperação total dos corpos d'águas, já que alguns compostos químicos, entre eles os metais pesados, permanecem nestes ambientes, principalmente nos sedimentos, por longos períodos de tempo

Recebido para publicação em 27/7/2007

Aceito para publicação em 17/7/2008 (002718)

${ }^{1}$ Instituto de Pesquisas Científicas - IPECI, Universidade Católica de Santos - UNISANTOS, Rua Dr. Carvalho de Mendonça, 144, CEP 11070-100, Santos - SP, Brasil,

E-mail: lgeraldo@unisantos.br

${ }^{*}$ A quem a correspondência deve ser enviada 
e, assim, podem estar sendo bioacumulados pela flora e fauna existentes nestes locais.

Os siris da Família Portunidae são muito comuns em regiões costeiras de climas tropicais, subtropicais e temperados. As espécies do gênero Callinectes sp. estão amplamente distribuídas nas regiões neotropicais e subtropicais e são muito importantes nas relações tróficas entre peixes e animais de fundos arenoso e lodoso. As espécies principais que ocorrem no litoral brasileiro são: Callinectes sapidus, C. danae, C. bocourti, C. ornatus, C. exasperatus, C. marginatus, C. similis sendo que na região da Baixada Santista, predominam as quatro primeiras espécies (MELLO, 1996).

A pesca do siri azul na região do município de Cubatão vem sendo feita principalmente pela população de baixa renda como um meio de subsistência. Grande parte é comercializada para restaurantes e bares da região, tornando importante a investigação dos teores de metais pesados nestes animais como uma forma de prevenção contra possíveis transferências destes poluentes para a população de uma forma geral.

O objetivo principal deste trabalho foi a determinação do teor de $\mathrm{Cd}, \mathrm{Pb}, \mathrm{Cr}, \mathrm{Cu}$ e $\mathrm{Zn}$ em quatro espécies de siris azuis(C. sapidus, C. danae, C. bocourti, C. ornatus) coletados ao longo do rio Cubatão, utilizando a técnica da Espectrometria de Absorção Atômica por Chama (FAAS). De especial interesse foi verificar a ocorrência de possíveis contaminações nestes animais e suas relações com as diferentes classes de tamanho, espécie e sexo dos indivíduos amostrados.

\section{Material e métodos}

As espécies de siris do gênero Callinectes sp. foram coletadas entre os anos de 2002 e 2004, nos períodos de estiagem e de maré baixa, ao longo do rio Cubatão por pescadores da região, utilizando armadilhas de captura do tipo puçá. Foram amostrados 144 indivíduos em várias locais, dentro de um trecho em torno de $8 \mathrm{~km}$ próximo ao estuário de Santos. Não houve preocupação com a localização exata, uma vez que os siris migram conforme as marés e salinidades. Os animais capturados foram acondicionados em caixas de isopor e no laboratório, armazenados em sacos plásticos, dentro de um freezer a uma temperatura em torno de $-20^{\circ} \mathrm{C}$, para posterior análise. Após o descongelamento à temperatura ambiente, foram pesados e selecionados quanto à espécie, utilizando uma chave de identificação específica sugerida por Mello (1996). A sexagem dos indivíduos foi feita pela observação da forma do abdômen de acordo com Negreiros-Fransozo e Fransozo (1997). As fêmeas jovens foram identificadas pelo pleon triangular, enquanto as fêmeas adultas, pelo pleon semicircular. Os machos possuem o abdômen muito estreito e afilado. Após serem biometrados, seguindo critérios padronizados para os Brachyura (MELLO, 1996), a carapaça do animal foi separada e as partes moles restantes pesadas para obtenção do peso úmido (fresco) total. A seguir, homogeneizou-se toda a parte mole da amostra em uma placa de Petri e procedeu-se a sua secagem em uma estufa a uma temperatura em torno de $150{ }^{\circ} \mathrm{C}$ por um período de tempo necessário para se obter uma diferença de peso menor que $1 \%$ nas sucessivas pesagens do material. Este procedimento permitiu determinar os pesos totais tanto na forma úmida como na forma seca para todas as amostras.

Para o preparo das amostras, seguiu-se a metodologia recomendada por Sawidis et al. (2001). Massas em torno de 0,5 g a 2,0 g de material seco (de acordo com o tamanho do animal) foram pesadas em uma balança digital Quimis $( \pm 0,0001 \mathrm{~g})$. $\mathrm{O}$ material foi transferido para um copo béquer de $50 \mathrm{~mL}$, acrescido de $5 \mathrm{~mL}$ de ácido nítrico concentrado (PA) por grama de material utilizado, e o conjunto, tapado com filme plástico de PVC com o objetivo de proteger a amostra e evitar a evaporação rápida do ácido. Após um período mínimo de 24 horas à temperatura ambiente, a amostra foi transferida para um bloco digestor (Digesdahl da Jundilab), equipado com um condensador de refluxo, iniciando-se o processo a $50^{\circ} \mathrm{C} \mathrm{e}$, lentamente, aumentando-se a temperatura até atingir $125^{\circ} \mathrm{C}$, na qual permaneceu por um período de tempo em torno de 6 horas. Em seguida, o resíduo líquido foi filtrado em papel filtro (Whattmann), transferido a um balão volumétrico de $25 \mathrm{~mL}$ e o volume completado com solução de ácido nítrico $2 \%$.

A determinação dos teores de metais pesados foi realizada utilizando-se um Espectrofotômetro de Absorção Atômica com Chama (FAAS) produzida por uma mistura de ar-acetileno, marca Perkin Elmer modelo A Analyst 100. As soluções padrões utilizadas na calibração do espectrômetro foram produzidas a partir de soluções estoques fornecidas pela Tec-Lab com concentrações da ordem de $1000( \pm 0,3 \%)$ ppm.

Para verificar a existência ou não de correlação entre os parâmetros estudados: massa, tamanho, sexo e concentração de metal pesado, foram utilizados dois programas estatísticos: SYSTAT e ANOVA. No programa SYSTAT foi empregada a rotina GLM (General Linear Model) com análise de resíduos, para o estudo das interações entre os parâmetros. O programa ANOVA (Analysis Of Variance) foi utilizado para verificar a ocorrência de similaridades ou não entre os parâmetros estudados.

\section{Resultados e discussão}

Neste estudo, foram analisados 144 amostras de siris do gênero Callinectes sp., com a seguinte distribuição nas 4 espécies: C. danae (91 ou 63\%); C. sapidus (33 ou 23\%); C. bocourti (15 ou 10,4\%); e C. ornatus ( 5 ou $3,4 \%$ ).

Considerando que o propósito principal deste trabalho era verificar os teores de metais pesados nas quatro espécies de siris azuis, normalmente utilizadas como forma de alimento pela população local, os resultados experimentais aqui apresentados referem-se à parte mole ou a todos os órgãos do animal (músculo, brânquias e hepatopâncreas) sem distinção.

A determinação dos teores de metais de interesse nas amostras de siris pelo FAAS foi feita, quando possível, em triplicata, e o resultado, para cada animal, foi obtido pela média das análises. Para o chumbo, cerca de $42 \%$ das amostras apresentaram resultados abaixo do limite de detecção do aparelho $(0,24 \mathrm{mg} \mathrm{Pb} / \mathrm{L})$. Desta forma, em todas as discussões relativas a este metal, foi adotada como valor representativo a mediana dos resultados. 


\subsection{Concentração de metal pesado versus tamanho dos animais}

Os exemplares de Callinectes sp. capturados apresentaram larguras no intervalo entre 7,0 e $15,3 \mathrm{~cm}$. A massa total dos animais incluindo a carapaça variou entre 16,2 e 238,3 g. Na Figura 1, é apresentado o comportamento da variação da massa corporal com a largura para todos os animais amostrados neste trabalho. As diferenças de massas observadas para indivíduos de mesmo tamanho podem ser explicadas pela ocorrência do processo de muda (troca de carapaça), um fenômeno que está associado ao crescimento destes animais. Durante este processo, o animal absorve água através do intestino que o auxilia a crescer, aumentando o seu volume, mas, não necessariamente, o seu tamanho (HICKMAN; ROBERTS; LARSON, 2004). Desta forma, um indivíduo nesta fase pode pesar muito mais que outro de mesmo tamanho, mas que já passou por esse processo de muda.

Os animais foram separados em 6 classes de largura, estando as 5 primeiras classes com diferença de $1 \mathrm{~cm}$ entre elas. A última classe, devido ao número menor de indivíduos, contém todos os exemplares com tamanhos maiores que $12,1 \mathrm{~cm}$. Desse modo, a classe 1 incluiu indivíduos entre 7,0 e 8,0 cm ( $\mathrm{n}=16)$; a classe 2 entre 8,1 e 9,0 cm ( $\mathrm{n}=26)$; a classe 3 entre 9,1 e 10,0 cm $(\mathrm{n}=31)$; a classe 4 entre 10,1 e $11,0 \mathrm{~cm}(\mathrm{n}=33)$; e a classe $5 \mathrm{com}$ indivíduos entre 11,1 e 12,0 $\mathrm{cm}(\mathrm{n}=13)$. A classe 6 incluiu os animais com larguras entre 12,1 e 15,3 cm $(\mathrm{n}=10)$. Só foram considerados para este estudo, os animais que apresentavam o espinho maior intacto $(\mathrm{n}=129)$; para as outras análises (sexo, espécie), todos os indivíduos foram incluídos $(\mathrm{n}=144)$.

Na Figura 2, é apresentado um gráfico do comportamento dos níveis de metais pesados obtidos neste trabalho, em função das classes de tamanho dos animais. Observa-se, na Figura 2 (a), que, apesar do chumbo apresentar uma razoável diminuição, os teores de cádmio e cromo permaneceram praticamente constantes em todo o intervalo das classes de largura. Estes metais são acumulados em função de suas concentrações no meio ambiente e, de acordo com o gráfico, os níveis encontrados não sofreram muita alteração.

No caso dos crustáceos, mais especificamente os siris, os metais pesados, quando em excesso, tendem a ser eliminados

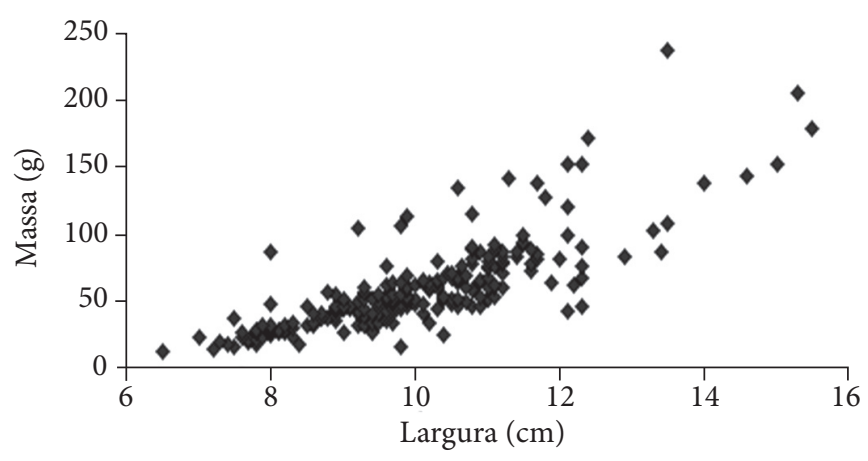

Figura 1. Variação da massa (g) corporal (material fresco com carapaça) em relação ao tamanho do siri amostrado (largura entre espinhos laterais). para o ambiente através de processos fisiológicos (excreção) ou durante o processo de muda. Keteles e Fleeger (2001), por exemplo, observaram que durante os períodos de intermuda e muda, os níveis de cádmio não se alteram significantemente $\mathrm{e}$ que, após a muda (ecdise), os níveis de cádmio diminuem. Isso sugere que, durante o processo de muda, o excesso de cádmio é eliminado juntamente com a carapaça (muda), ficando praticamente constante durante o crescimento do animal.

De acordo com a Figura 2b, observa-se que os menores exemplares apresentam as maiores concentrações de metais e, a partir da $2^{\text {a }}$ classe, os níveis de $\mathrm{Cu}$ e $\mathrm{Zn}$ tendem a diminuir, mas de forma um pouco mais intensa para o cobre. Após aproximadamente a metade do intervalo entre 10,1 e $11,0 \mathrm{~cm}$, o comportamento tende a inverter-se para o caso do cobre. Os elementos zinco e cobre são micronutrientes essenciais para crustáceos decápodos. O primeiro é usado como ativador de sistemas enzimáticos, enquanto o cobre é utilizado como parte integrante do pigmento respiratório hemocianina (RAINBOW, 1997). Isso pode explicar as diferenças de concentrações entre jovens e adultos em função de suas necessidades metabólicas diferentes.
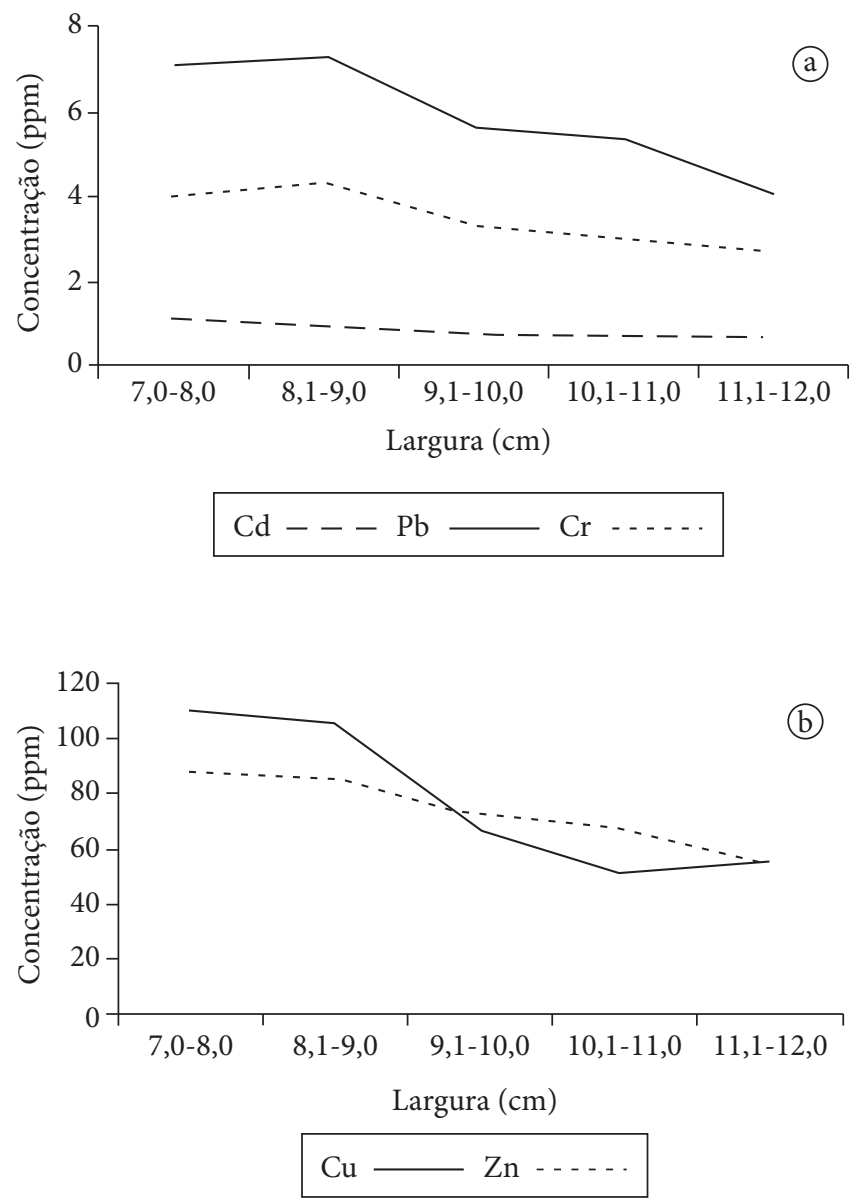

Figura 2. Variação das concentrações médias de metais (em ppm de peso seco), a) $\mathrm{Cd}, \mathrm{Pb}, \mathrm{Cr}$; e b) $\mathrm{Cu}$ e $\mathrm{Zn}$, em relação às diferentes classes de largura $(\mathrm{cm})$ dos animais. No caso do chumbo, foi utilizada a mediana das leituras. 
De acordo com Engel e Brouwer (1991), a concentração de zinco e cobre decresce durante a ecdise ou muda e permanece relativamente constante durante o período de pós-muda, quando então a carapaça ainda não está rígida. A diminuição na concentração de metal nesse período é causada pela absorção intensa de água pelo animal que o auxilia no processo de crescimento corporal. Durante essa fase de pós-muda, o estoque de cobre é utilizado para sintetizar hemocianina e auxiliar no processo de endurecimento do exoesqueleto (KETELES; FLEEGER, 2001).

Era de se esperar que animais maiores acumulassem maiores quantidades de metais pesados em seus organismos em função do tempo mais longo ao qual estiveram expostos, mas foi observado o contrário. Para os crustáceos, animais jovens e menores tendem a mudar mais vezes que os animais adultos. Da fase de zoea até a fase jovem mudam cerca de 7 vezes em 7 semanas, enquanto, na fase adulta, realizam em torno de 20 mudas no período de 3 anos (CAMERON, 1985). Essas mudas ocorrem em função da maior atividade metabólica que os animais possuem na fase de crescimento. Devido a este processo, ingerem uma quantidade maior de alimentos e, conseqüentemente, mais contaminantes, caso estes estejam presentes (MANTELATTO; CHRISTOFOLETTI, 2001).

De acordo com Ferrer et al. (2006), jovens caranguejos são considerados agentes potencialmente perigosos para a transferência de contaminantes ao longo da cadeia trófica (como alimento para peixes e pássaros), principalmente devido a sua elevada resistência e a sua maior capacidade de bioacumular metais pesados em seus tecidos em relação aos indivíduos adultos. Entretanto, neste trabalho não foram observadas diferenças significativas (ao nível $\mathrm{p}<0,5$ ) para os teores de metais em relação ao tamanho dos indivíduos.

\subsection{Concentração de metal pesado versus espécie de siri azul}

Na Tabela 1, são listados os teores médios de metais pesados obtidos para as 4 espécies de siri azul estudadas neste trabalho. Foi escolhida a apresentação dos resultados em peso úmido (fresco) uma vez que os limites sugeridos pelas agências de saúde são expressos nesta forma. A média das razões entre todos os pesos totais determinados para as amostras no estado úmido (fresco) com os respectivos pesos no estado seco foi de $4,9 \pm 1,7$. De acordo com os testes estatísticos efetuados, verifica-se que, com exceção do valor para o chumbo, expresso em mediana, todos os outros resultados foram mais altos para a espécie Callinectes sapidus a um nível de significância de $\mathrm{p}<0,01$ para o Cd, $\mathrm{p}<0,05$ para o $\mathrm{Cu}, \mathrm{p}<0,05$ para o $\mathrm{Cr}$ e $\mathrm{p}<0,01$ para o $\mathrm{Zn}$. O teste estatístico não foi aplicado no caso do chumbo, por não ter sido possível a obtenção de um valor médio para os resultados.

Tabela 1. Concentração média $( \pm 1 \sigma)$ de metal pesado (em ppm de peso úmido) para as quatro espécies de siri azul estudadas neste trabalho.

\begin{tabular}{lcccc}
\hline \multicolumn{1}{c}{ Metal } & C. bocourti & C. ornatus & C. sapidus & C. danae \\
\hline Cádmio & $0,137 \pm 0,088$ & $0,20 \pm 0,12$ & $0,22 \pm 0,13$ & $0,124 \pm 0,098$ \\
Cromo & $0,71 \pm 0,43$ & $0,53 \pm 0,26$ & $0,82 \pm 0,45$ & $0,65 \pm 0,33$ \\
Chumbo $^{*}$ & $0,612 \pm 0,067$ & $1,51 \pm 0,16$ & $1,06 \pm 0,11$ & $1,10 \pm 0,12$ \\
Cobre & $15 \pm 13$ & $17 \pm 16$ & $18 \pm 10$ & $14 \pm 11$ \\
Zinco & $16 \pm 11$ & $14,9 \pm 9,8$ & $20 \pm 12$ & $12,0 \pm 7,3$ \\
\hline
\end{tabular}

${ }^{\star}$ Mediana dos resultados.
Diferenças entre os níveis de metais pesados encontrados em espécies do gênero Callinectes podem ser explicadas em virtude das possíveis estratégias de bioacumulação, itens alimentares e distribuição espaço-temporal praticadas por cada espécie. Mudanças na salinidade também afetam a biodisponibilidade e conseqüente absorção de metais-traço (ENGEL; BROUWER; MERCALDO-ALLEN, 2001). Um outro fator que tem sido também utilizado para explicar diferenças de concentração de metais pesados entre as espécies estudadas está relacionado à presença de uma maior ou menor quantidade de metalotioneina (proteína de baixo peso molecular). Esta proteína tem como funções a fixação, controle e regulação de elementos-traço como zinco e cobre bem como a neutralização de níveis tóxicos produzidos, principalmente, pela absorção de cádmio e mercúrio provenientes de ambientes estressados (2000). Martin-Diaz et al. (2005), trabalhando com Carcinus maenas, observaram que os metais pesados eram bioacumulados de maneiras diferentes em diferentes tecidos e que a indução da metalotioneina em tecidos do hepatopâncreas, não se comportava da mesma forma quando expostos aos metais pesados de maneira isolada ou combinada.

\subsection{Concentração de metal pesado versus sexo dos animais amostrados}

Foram analisados 120 machos e 24 fêmeas de siri azul num total de 144 indivíduos, e os valores médios para os teores de metais pesados obtidos são apresentados na Tabela 2. Pode-se observar que, de uma maneira geral, existe uma tendência das fêmeas concentrarem uma maior quantidade de metais pesados. Entretanto, de acordo com os testes estatísticos realizados, apenas para o caso do $\mathrm{Cu}$ é que as fêmeas apresentaram um teor médio significativamente maior que os machos a um nível $\mathrm{p}<0,05$.

O pequeno número de fêmeas das quatro espécies analisado neste trabalho pode ser explicado em função do comportamento desses animais. Normalmente, as fêmeas de siri azul (Callinectes sp.) encontram-se fora do estuário em águas de salinidade mais alta, juntamente com as larvas, dificultando assim a sua captura em ambientes de estuário. Por outro lado, os machos e os mais jovens, usualmente, concentram-se no estuário e em áreas de manguezais de baixa salinidade (CHACUR; NEGREIROS-FRANSOZO, 2001).

Tabela 2. Valores médios da concentração de metal pesado (em ppm de peso úmido) para Machos (M) e Fêmeas (F) de siri azul do gênero Callinectes sem distinção quanto à espécie.

\begin{tabular}{ccc}
\hline Metal & Sexo & Concentração $( \pm 1 \sigma)$ \\
\hline $\mathrm{Zn}$ & $\mathrm{F}$ & $14,9 \pm 6,5$ \\
& $\mathrm{M}$ & $13,9 \pm 2,2$ \\
$\mathrm{Cu}$ & $\mathrm{F}$ & $22,5 \pm 1,5$ \\
& $\mathrm{M}$ & $13,3 \pm 5,9$ \\
$\mathrm{Cd}$ & $\mathrm{F}$ & $0,184 \pm 0,061$ \\
& $\mathrm{M}$ & $0,135 \pm 0,035$ \\
$\mathrm{~Pb}^{*}$ & $\mathrm{~F}$ & $1,35 \pm 0,37$ \\
& $\mathrm{M}$ & $0,96 \pm 0,35$ \\
$\mathrm{Cr}$ & $\mathrm{F}$ & $0,75 \pm 0,35$ \\
& $\mathrm{M}$ & $0,653 \pm 0,090$ \\
\hline
\end{tabular}

${ }^{\star}$ Mediana dos resultados. 
Macfarlane et al. (2000), trabalhando com Heloecius cordiformis (caranguejo terrestre), observaram que, quando animais de sexos diferentes são encontrados em um mesmo local, a tendência é de que as fêmeas acumulem mais cobre e zinco em relação aos machos, como uma função da maturidade sexual e não do tamanho. Entretanto, este comportamento foi observado apenas para o caso do cobre, independentemente da maturação sexual ou tamanho. Como se pode observar na Tabela 2, a concentração de zinco entre machos e fêmeas é praticamente a mesma, dentro das incertezas experimentais, o mesmo ocorrendo no caso do cromo. Ainda, de acordo com os mesmos autores, a concentração de chumbo em Heloecius cordiformis pode ser dependente do tamanho apenas para machos, ou seja, com os machos pequenos acumulando mais do que os machos maiores. Contudo, no presente trabalho, este comportamento com relação ao chumbo foi observado tanto para os machos como para as fêmeas. Isso pode ser explicado em função do metabolismo mais acelerado e das várias mudas que ocorrem para o crescimento dos jovens, como já foi discutido anteriormente. Além disso, a similaridade na dieta entre machos e fêmeas (MANTELATTO, 2000), bem como a dieta diferenciada entre jovens e adultos (MANTELATTO; CHRISTOFOLETTI, 2001), podem explicar essas diferenças. Isso quer dizer que, a similaridade ou diferença nas dietas, depende das diferentes necessidades metabólicas em função da idade (jovem e adulto) e algumas vezes da maturidade sexual das fêmeas para a produção do vitelo presente no ovo.

O comportamento de maior bioacumulação de metais pesados em caranguejos e siris pelas fêmeas em relação aos machos também foi observada por outros autores (MANTELATTO; CHRISTOFOLETTI, 2001; MACFARLANE; BOOTH; BROWN, 2000; COSTA; NEGREIROS-FRANSOZO, 1998). Mantelatto (2000), trabalhando com C. ornatus na Baía de Ubatuba, observou que existe uma similaridade na dieta entre machos e fêmeas, provavelmente, devido à ocupação do mesmo habitat durante grande parte do ciclo de vida. Entretanto, Mantelatto e Christofoletti (2001), observaram pequenas diferenças com relação à dieta entre os sexos e entre fêmeas ovígeras e não ovígeras. Fêmeas ovígeras se alimentavam menos do que as populações de machos e de fêmeas não ovígeras e sua preferência alimentar maior estava baseada em animais de tecidos macios como peixes, lulas e camarões.

Campbell et al. (2005), trabalhando com cobras aquáticas (Nerodia sipedon), observaram que as fêmeas eram significantemente maiores do que os machos e, conseqüentemente, era de se esperar concentrações maiores de metais nas fêmeas. Entretanto, seus resultados mostraram que a concentração de metal não parece se alterar entre os sexos e, de acordo com os autores, este fato talvez esteja relacionado à transferência materna do metal para os ovos.

\subsection{Comparação dos teores de metais pesados obtidos para as quatro espécies de siris azuis com valores recomendados por agências de saúde}

Os intervalos de valores médios obtidos neste trabalho para as 4 espécies de siris azuis são comparados na Tabela 3 com os limites estabelecidos pelo Ministério da Saúde (2003), com os valores re-
Tabela 3. Intervalo dos valores médios (em ppm de peso úmido) obtidos neste trabalho para as 4 espécies de siris azuis, comparados com os limites máximos recomendados por organizações de saúde.

\begin{tabular}{lcccr}
\hline \multicolumn{1}{c}{ Metal } & $\begin{array}{c}\text { FAO/WHO } \\
(1997 ; 2000)\end{array}$ & $\begin{array}{c}\text { Brasil } \\
(2006)\end{array}$ & $\begin{array}{c}\text { EPA } \\
(1996)\end{array}$ & \multicolumn{1}{c}{$\begin{array}{c}\text { Este } \\
\text { trabalho }\end{array}$} \\
\hline Cádmio & 1,0 & 1,0 & $>2,0$ & $0,124-0,22$ \\
Cromo total & 12 & 0,1 & $0,1-0,9$ & $0,53-0,82$ \\
Chumbo & 2,0 & 2,0 & $0,1-0,8$ & ${ }^{\star} 0,612-1,51$ \\
Cobre & 30,0 & 30,0 & $1,0-20,0$ & $14,0-18,0$ \\
Zinco & 50,0 & 50,0 & $10,0-30,0$ & $12,0-20,0$ \\
\hline
\end{tabular}

${ }^{*}$ Mediana dos resultados.

comendados pela $\mathrm{FAO} / \mathrm{WHO}(1997 ; 2000)$ e com os intervalos de concentrações considerados como normais pela EPA (1996).

Observa-se na Tabela 3 que, com exceção do cromo, os valores médios obtidos para todos os outros metais analisados neste trabalho estão, em geral, abaixo dos limites máximos recomendados pelas Agências de Saúde para as 4 espécies e, portanto, em nível de consumo, os siris da região do manguezal do rio Cubatão podem ser classificados como ainda não contaminados. No caso do cromo, apesar de ter sido obtido um intervalo para os teores médios acima do limite estabelecido pela legislação brasileira para qualquer tipo de alimento, observa-se que o resultado está dentro do intervalo considerado normal pela EPA para animais do tipo crustáceos ou moluscos e muito abaixo do limite máximo recomendado pela FAO/WHO. No caso do chumbo, as medianas de 3 espécies (C. ornatus, C. sapidus, C. danae) excederam o limite considerado normal pela EPA para moluscos e crustáceos, mas mesmo assim, estão abaixo dos limites estabelecidos pela legislação brasileira e pela $\mathrm{FAO} / \mathrm{WHO}$.

\section{Conclusões}

Neste trabalho, foram determinados os teores totais de $\mathrm{Pb}, \mathrm{Cd}, \mathrm{Cr}, \mathrm{Cu}$ e $\mathrm{Zn}$ em amostras de 4 espécies de siris azuis do genêro Callinectes sp., coletadas ao longo do rio Cubatão, utilizando a técnica da espectrometria de absorção atômica por chama. De especial interesse foi verificar a ocorrência de possíveis contaminações nestes animais e suas relações com as diferentes classes de tamanho, espécie e sexo dos indivíduos amostrados.

Apesar das amostragens terem sido efetuadas entre os anos de 2002 e 2004, não foi possível identificar uma variação temporal da concentração de metais pesados nestes animais.

De acordo com os resultados obtidos, não foram observadas diferenças significativas (ao nível $\mathrm{p}<0,5$ ) para os teores de metais pesados em relação ao tamanho dos indivíduos amostrados. Pelos testes estatísticos efetuados, verifica-se que, com exceção do valor para o chumbo, expresso em mediana, todos os outros resultados foram mais altos para a espécie Callinectes sapidus a um nível de significância de $\mathrm{p}<0,01$ para o Cd, $\mathrm{p}<0,05$ para o $\mathrm{Cu}, \mathrm{p}<0,05$ para o $\mathrm{Cr}$ e $\mathrm{p}<0,01$ para o $\mathrm{Zn}$. Quanto ao sexo dos animais, observou-se que, somente para o caso do $\mathrm{Cu}$, as fêmeas apresentaram teores médios significativamente maiores que os machos a um nível $\mathrm{p}<0,05$. 
Apenas no caso do Cr, o intervalo de valores médios obtidos para as 4 espécies ficou acima do limite estabelecido pela legislação brasileira para qualquer tipo de alimento. Entretanto, este resultado está ainda dentro do intervalo considerado normal pela EPA para animais do tipo crustáceos ou moluscos e muito abaixo do limite máximo recomendado pela $\mathrm{FAO} / \mathrm{WHO}$. Desta forma, as 4 espécies de siris azuis da região do manguezal do rio Cubatão, podem ser consideradas, para consumo, como não contaminadas em relação aos metais pesados estudados neste trabalho.

\section{Referências bibliográficas}

BRASIL. Portaria ${ }^{\circ}$. 685/98. Aprova o Regulamento Técnico: Princípios Gerais para o Estabelecimento de Níveis Máximos de Contaminantes Químicos em Alimentos e seu Anexo: Limites máximos de tolerância para contaminantes inorgânicos. Complementa e faz algumas modificações no Decreto Lei no 55.871 , de 26 de março de 1965. Disponível em: <http://e-legis.bvs.br/leisref/public/showAct. php?id=90 >. Acesso em: mar. 2003.

CAMERON, J. Molting in the blue crabs. Scientific American, v. 52, n. 1, p. 102-109, 1985.

CAMPBELL, K. R.; CAMPBELL, T. S.; BURGER J. Heavy metal concentrations in north water snakes (Nerodia sipedo) from east fork poplar creek and the little river, east Tennessee, USA. Archives of Environmental Contamination Toxicology, v. 49, n. 2, p. 239-48, 2005.

CETESB - COMPANHIA DE TECNOLOGIA DE SANEAMENTO AMBIENTAL. Secretaria do Meio Ambiente do Governo do Estado de São Paulo. Contaminantes na Bacia do rio Cubatão e seus Reflexos. São Paulo, 1990. 81p. (RelatórioTécnico).

CETESB - Companhia de Tecnologia de Saneamento Ambiental. Secretaria do Meio Ambiente do Governo do Estado de São Paulo. Sistema Estuarino de Santos e São Vicente. São Paulo, v. 1-2, 2001. 178p. (RelatórioTécnico).

CHACUR, M. M.; NEGREIROS-FRANSOZO, M. L. Spatial and seasonal distributions of Callinectes danae (Decapoda, Portunidae) in Ubatuba Bay, São Paulo, Brazil. Journal of Crustacean Biology, v. 21, n. 2, p. 414-425, 2001.

COSTA, T. M.; NEGREIROS-FRANSOZO, M. L. The reproductive cycle of Callinectes danae Smith, 1869 (Decapoda, Portunidae) in the Ubatuba region, Brazil. Crustaceana, v. 71, n. 6, p. 615-627, 1998.

ENGEL, D. W.; BROUWER, M. Short metallothionein and cooper changes in blue crabs at ecdysis. Biological Bulletin, v. 180, n. 3, p. 447-452, 1991.

ENGEL, D. W.; BROUWER, M.; MERCALDO-ALLEN, R. Effects of molting and environmental factors on trace metal bodyburdens and hemocyanin concentrations in the American lobster, Homarus americanus. Marine Environmental Research, v. 52, n. 3, p. 257-269, 2001.

EPA - Environmental Protection Agency. Guidance for Assessing Chemical Contaminant Data for Use in Fish Advisories. Fish Sampling and Analysis. EPA 823-R-93-002. In: HEINEN, J. M. Water Quality Criteria, Uptake, Bioaccumulation, and Public Health Considerations for Chemicals of Possible Concern in West Virginia Mine Waters Used for Culture of Rainbow Trout. 1995. Washington, D.C., March 1996.

EPA. Environmental Protection Agency. Guidance for Assessing Chemical Contaminant Data for Use in Fish Advisories, v. 2. Risk Assessment and Fish Consumption Limits. EPA 823-B-94-004. In: HEINEN, J. M. Water Quality Criteria, Uptake, Bioaccumulation, and Public Health Considerations for Chemicals of Possible Concern in West Virginia Mine Waters Used for Culture of Rainbow Trout. 1995. Washington, D.C., March 1996.
FAO/WHO - FOOD AND AGRICULTURE ORGANIZATION/ WORLD HEALTH ORGANIZATION. Food consumption and exposure assessment of chemicals. Report of a FAO/WHO consultation, 10-14 February. Geneva, Switzerland, 1997.

FAO/WHO - FOOD AND AGRICULTURE ORGANIZATION/ WORLD HEALTH ORGANIZATION. Evaluation of certain food additives and contaminants for lead and methylmercury. In: Report of the Joint FAO/WHO Expert Committee on Food Additives, $53^{\text {rd }}$. Technical Report Series 896. Geneva, Switzerland, 1999.

FAO/WHO - FOOD AND AGRICULTUREORGANIZATION/WORLD HEALTH ORGANIZATION. Evaluation of certain food additives and contaminants for cadmium and methylmercury. In: Report of the Joint FAO/WHO Expert Committee on Food Additives, $55^{\text {th }}$. Technical Report Series 901. Geneva, Switzerland. 2000.

FERRER, L. et al. Acute toxicities of four metals on the early life stages of the crab Chasmagnathus granulate from Bahia Blanca, Argentina. Ecotoxicology and Environment Safety, v. 65, n. 2, p. 209-217, 2006.

HICKMAN Jr., C. P.; ROBERTS, L. S.; LARSON, A. Princípios Integrados de Zoologia. 11 ed. Rio de Janeiro: Guanabara Koogan, 2004.

KETELES, K. A.; FLEEGER, J. W. The contribution of ecdysis to the fate of cooper, zinc and cadamium in grass shrimp, Palaemonetes pugio Holthius. Marine Pollution Bulletin, v. 42, n. 12, p. 1397-1402, 2001.

LEGRAS, S. et al. Changes in metallothionein concentrations in response to variation in natural factors(salinity, sex, weight) and metal contamination in crabs from a metal rich estuary. Journal of Experimental Marine Biology and Ecology, v. 246, n. 2, p. 259-279, 2000.

MACFARLANE, G. R.; BOOTH, D. J.; BROWN, K. R. The semaphore crab, Heloecius cordiformis: bioindication potential for heavy metals in estuarine systems. Aquatic Toxicology, v. 50, n. 3, p. 153-166, 2000.

MANTELATTO, F. L. M. Allocation of the portunidae crab Callinectes ornatus (Decapoda, Brachyura) in Ubatuba Bay, northern coast of São Paulo State, Brazil. Crustaceana, v. 12, n. 2, p. 431-443, 2000.

MANTELATTO, F. L. M.; CHRISTOFOLETTI, R. A. Natural feeding activity of the crab Callinectes ornatus (Portunidae) in Ubatuba Bay (São Paulo, Brazil): Influence of season, sex, size and the molt stage. Marine Biology, v. 138, n. 3, p. 585-594, 2001.

MARKERT, B. Distribution and Biogeochemistry of Inorganic Chemicals in the Environment. In: SCHÜÜRMANN, G. and MARKERT, B. (eds.). Ecotoxicology. Heydelberg, Germany: John Wiley and Sons. Inc and Spektrum Akademischer Verlag, 1998. Part 2, p. 165-199.

MARTIN-DIAZ, M. L. etal. An integrated approach using bioaccumulation and biomarker measurements in female shore crab, Carcinus maenas. Chemosphere, v. 58, n. 5, p. 615-626, 2005.

MELLO, G. A. S. Manual de Identificação dos Brachyura (caranguejos e siris) do Litoral Brasileiro. São Paulo: Plêiade/FAPESP - Fundação de Amparo a Pesquisa do Estado de São Paulo, 1996. 604p.

NEGREIROS-FRANSOZO, H. L.; FRANSOZO, A. Avaliação preliminar da biologia de C. ornatus Ordway, 1863 e C. danae Smith, 1869 (crustácea, Decapoda) na região de Ubatuba-SP, Brazil. In: Simpósio de Ecossistemas da Costa Brasileira: subsídios a um gerenciamento ambiental, 3, 1994, São Paulo. Anais... São Paulo: ACIESP Pub.87, n. 1, p. 122-130, 1994.

RAINBOW, P. S. Ecophysiology of trace metal uptake in crustaceans. Estuarine, Coastal and Shelf Science., v. 44, n. 2, p. 167-175, 1997.

SAWIDIS, T et al. A Study of Metal Distribution from Lignite Fuels Using Trees as Biological Monitors. Ecotoxicology and Environmental Safety, v. 48, n. 1, p. 27-35, 2001.

WHO-World Health Organization. Report of the Join FAO/WHO Expert Committee of Food Additives, $16^{\text {th }}$. Technical report series 50. Geneva, Switzerland, 1972. 